\title{
Neo-modified Koyanagi technique for severe hypospadias with one-stage sealed Y-shaped penis foreskin vascular protection surgery
}

Jiancheng $\mathrm{Zu}^{1}$, Yifu Chen ${ }^{1}$, Yu Liu ${ }^{1}$, Tianqu $\mathrm{He}^{1}$, yanling wang ${ }^{2}$, Yuee $\mathrm{Zu}^{1}$, and Xiangwen Peng 3

${ }^{1}$ Hunan Children's Hospital

${ }^{2}$ Wuhan University

${ }^{3}$ Hunan Normal University

October 4, 2021

\begin{abstract}
A total of 89 children had their urinary catheters removed 4 weeks after the operation. The children, diagnosed with urine leakage, were successfully repaired after the leakage occurred one year later at one time.The one-time success rate of this operation was $87.6 \%$ and the incidence of the urethral fistula was $12.6 \%$
\end{abstract}

\section{Article category:technical note}

\section{Title}

Neo-modified Koyanagi technique for severe hypospadias with one-stage sealed Y-shaped penis foreskin vascular protection surgery

\section{Author names and affiliations}

Jiancheng Zu ${ }^{1}$, Yifu Chen ${ }^{1}$, Yu Liu ${ }^{1}$, Tianqu $\mathrm{He}^{1}$, Yanling Wang ${ }^{2}$, Yuee $\mathrm{Zu}^{2 *}$, Xiangwen Peng ${ }^{2 *}$

${ }^{1}$ Hunan Children's Hospital, 86\# Ziyuan Road, Changsha City, Hunan Province, P.R.China, Changsha City, Hunan Province, P.R.China, 410000

${ }^{2}$ Changsha Hospital for Maternal and Child Health Care of Hunan Normal University,416\# Chengnan East Road, Yuhua District, Changsha City, Hunan Province, P.R.China, 410000

\section{Conflicts of interest statement}

The authors declare that they have no conflict of interest.

\section{* Corresponding author:}

Xiangwen Peng (Email: pxw1237@163.com; mailing address:416\# Chengnan East Road, Yuhua District, Changsha City, Hunan Province, P.R.China, 410000; telephone:17665082951; fax numbers:0731-84134539);

Yuee Zu (Email:1163605177@qq.com;mailing address:Hunan Children's Hospital, 86\# Ziyuan Road, Changsha City, Hunan Province, P.R.China; telephone:13307499673; fax numbers:0731-85356010)

\section{Running title}

Neo-modified technique for severe hypospadias

Key clinical message 
A total of 89 children had their urinary catheters removed 4 weeks after the operation. All children had reviewed at least once a year for 3 consecutive years. There are 11 patients with urine leakage, who occurred after the operation. The children, diagnosed with urine leakage, were successfully repaired after the leakage occurred one year later at one time. There was no urethral stricture after an operation. The one-time success rate of this operation was $87.6 \%(78 / 89)$ and the incidence of the urethral fistula was $12.6 \%(11 / 89)$.

\section{Abstract}

Purpose: Proximal hypospadias defects represent the most challenging of maintaining blood supply to the flap, which eventually leads to a high rate of complications. We modify a sealed Y-shaped penis foreskin vascular protection technique, which can repair the urethra in a single stage.

Methods: Cut the inner plate of the foreskin along the coronal sulcus, cut both sides of the urethral plate as deep as Buck's fascia. The "Y"-shaped foreskin flaps on both sides of the mouth that are continuous with the urethral plate are sutured to form a new urethral skin tube. The urethral skin tube is turned to the ventral side, and the foreskin is reshaped and sutured.

Results: A total of 89 children had their urinary catheters removed 4 weeks after the operation. All children had reviewed at least once a year for 3 consecutive years. There are 11 patients with urine leakage, who occurred after the operation. The children, diagnosed with urine leakage, were successfully repaired after the leakage occurred one year later at one time. There was no urethral stricture after an operation. The one-time success rate of this operation was $87.6 \%$ (78/89) and the incidence of the urethral fistula was $12.6 \%$ $(11 / 89)$.

Conclusion: The results showed that sealed Y-shaped penis foreskin vascular protection surgery had a safer and higher operation rate than the traditional hypospadias repair technique. Modifying the Koyanagi repair by our improved Koyanagi hypospadias repair is an excellent technique with relatively low complication rates.

Keywords: Koyanagi technique,Y-shaped penis,surgery,Proximal hypospadias

\section{Introduction}

The Koyanagi technique has received a lot of attention for its logical flap design since its development $[1 ; 2]$. Many modifications of the Koyanagi technique have been introduced because of its high complication rate[3; $4 ; 5 ; 6]$. But the complication rate didn't drop much. We believe that the high complication rate is mainly due to the disruption of blood supply to the flap. Here, we development a modified Koyanagi technique that improves the blood supply of the flap for single stage repair of severe hypospadias, thereby greatly reducing the occurrence of complications. Similar to the Koyanagi technique, the urethral plate was recreated using foreskin, but then a sealed Y-shaped incision was made on the original and recreated urethral plate. Different from the Koyanagi technique where the foreskin is cut, it only be cut to the skin layer, we divide the foreskin into the epidermal layer and the subcutaneous tissue layer. The epidermis is cut off, but the subcutaneous tissue is separated and the vascular system is protected. In this way, the blood supply of the flap is intact, reducing operation failures caused by avascular necrosis of the flap.

\section{Operative Technique}

Preoperative examination: chromosome, scrotum, groin, kidney, ureter, bladder, heart color ultrasound, liver and kidney function, electrocardiogram. Wash vulva area with running water 12 hours before surgery.Fasting 6-8 hours preoperatively and cefuroxime 30 minutes preoperatively.

In the supine position, $0.05 \%$ complexed iodine disinfects the skin, $0.05 \%$ complexed iodine disinfects the foreskin and urethral opening, F8 catheter is smoothly inserted into the bladder and left in, and $0.05 \%$ complexed iodine disinfects the foreskin. Suture No. 1 silk thread on the dorsal side of the glans as a traction line. Cut the inner plate of the foreskin to the albuginea layer at a distance of $0.5 \mathrm{~cm}$ from the coronal sulcus, and then sneak along the albuginea layer to the root of the penis(Fig1.B-C). Do a penile erection test with salt water to check that the flexion of the penis has been corrected. Measure the distance 
between the head of the penis and the ectopic urethral orifice as $4.1 \mathrm{~cm}$. Take the joint flaps of the penis and scrotum with a continuous width of about $6 \mathrm{~mm}$ along the inner foreskin on both sides of the ectopic urethral orifice, and cut the foreskin skin (the skin cannot be cut) at 12 o'clock on the back of the penis. (the skin cannot be cut), free the subcutaneous skin, pay attention to protect the blood supply of the skin flap, free the urethral plate along both sides of the ectopic urethral orifice, and the skin flap will become a sealed "Y" shape, and the inner edges of the two arms of the sealed Y-shaped skin flap are intermittently sutured(Fig1.D-J). Make the sealed Y-shaped skin flap into a rectangular skin flap. The outer edge of the rectangular skin flap is intermittently sutured along the urethral catheter to form a urethral skin tube. A penis-sized hole is bluntly separated from the center of the urethral skin tube, and the penis is passed through the small Pull out the hole to transfer the urethral tube to the ventral side of the penis. Separate a tunnel into the cavernous tissue $0.5 \mathrm{~cm}$ below the ventral coronal sulcus of the head of the penis, cut the ventral side of the tunnel, and connect the distal end of the urethral tube to the head of the penis. 6-0 intermittent suture with absorbable thread to form a new urethral opening. An arc incision was made on the upper pole of the scrotum, the penis was lifted, the penile scrotal incomplete transposition was corrected, the excess foreskin was transferred from the dorsal side to the ventral side, and the new urethra was covered with intermittent suture with 6-0 absorbable thread(Fig1.K-N). Wrap the penile wound with petroleum jelly gauze, press the scrotum wound with petroleum jelly, use cefuroxime for 5 days after the operation, and remove the petroleum jelly gauze one week after the operation. The urinary catheter was pulled out 4 weeks after the operation, and the urination was observed.

Tips: 1. foreskin flap can only be cut to the skin layer, and the 12 o'clock dorsal foreskin incision can only cut the skin layer.

2. A vascular pedicle can be used to cover the urethra.

3. The thickness of tunnel and subcutaneous should not exceed $1.5 \mathrm{~mm}$.

\section{Results}

From 2014-2017, 29 cases of perineal hypospadias and 60 cases of scrotal hypospadias have been done in my institutions. The ages of the patients ranged from 1 to 10 years (mean=4 years). All children had their urinary catheters removed 4 weeks after the operation. All children had their urinary catheters removed 1,3 , and 6 months, and then reviewed at least once a year for 3 consecutive years. Nine cases of urine leakage occurred within 3 days after the operation. The leakage orifices were all located at the junction of the penis ventral penis and scrotum. The diameter of the leakage orifice was about $2-5 \mathrm{~mm}$. One case had urine leakage 3 months after the operation, and 1 case was 5 after the operation. Urine leakage occurred in the first month, and the leakage opening was located in the middle of the ventral side of the penis, with a diameter of about $1 \mathrm{~mm}$. It is considered that the needle eye caused the leakage of urine after the absorbable thread was absorbed. One case of urine leakage was caused by skin infection in 1 year after the operation. All the children with urine leakage were repaired after the leakage occurred one year later, and the leakage was successfully repaired at one time. There was no urethral stricture after operation. The one-time success rate of this operation was $87.6 \%(78 / 89)$ and the incidence of urethral fistula was $12.6 \%(11 / 89)$.

\section{Discussion}

The patient's repaired of proximal hypospadias surgery was extremely challenging, because of having many surgical complications. At present, the more popular stage I surgery is: the urethral plate is cut off to straighten the penis to correct the curvature of the penis, and the urethra is formed by the cross-cutting island-shaped foreskin flap[7]. Other modern techniques include: Thierch-Duplay, Onlay , and stage II surgery[8; 9]. In 1975, Reddy first described the urethral plate incision and tube urethroplasty[10]. In 1994, Snodgrass et al. Popularization, but recent long-term follow-up studies have shown that: for proximal hypospadias, most children have functional urinary flow obstruction and decreased urinary flow rate after the operation[11]. Since most children with proximal hypospadias have severe penile curvature, the fibrous tissue of the urethral plate needs to be removed for correction. Therefore, the urethral plate preservation surgery (Thierch-Duplay, Onlay, urethral plate incision and roll tube urethroplasty) It is not an ideal surgical 
method. Stage I urethroplasty with continuous sealed Y skin flap of penis and scrotum can sharply peel off the urethral plate, remove the fiber cord, completely straighten the penis, and enable stage I urethroplasty.

Since 2000, more in-depth research has been conducted on both sides of the base of the urethral orifice and the inner foreskin flap on the back for repairing proximal hypospadias. Stage I urethroplasty with continuous sealed Y skin flap of penis and scrotum is a simple technique. It has six advantages. Firstly, the material of the new urethral flap is sufficient. The new urethroplasty flap used in this operation is continuous from the urethral opening to the dorsal foreskin inner plate. The length of the flap is exactly the distance from the ectopic urethral orifice to the tip of the penis. Do not measure the length of the flap. Secondly, preserve the original natural anatomical structure. It is currently believed that the urethral plate is a natural urethroplasty structure of hypospadias, and the inner plate of the foreskin and the urethra come from the same germ layer during embryonic development. Utilizing the inner foreskin and urethral plates on both sides of the urethra orifice as the new urethroplasty material, it is most in line with the natural physiological anatomy of hypospadias; at the same time, the original axial blood supply of the entire skin flap of the new urethra is almost unchanged, which is conducive to wound healing and surgery. After the complications are repaired again, the success rate of the reoperation in this group is higher; Thirdly , make the bilateral scrotum suture to eliminate the scrotal division and translocation. Fourthly, There is no anastomosis in the new urethra. The joint of the original urethral orifice of the operation is formed by a rolled tube urethral plate. There is no need for circular anastomosis, which can significantly reduce complications such as anastomotic stenosis; Fifthly, the success rate is high. Preserve the blood supply of the distal foreskin, so that the flaps will not be too long and poor blood supply, especially with the scrotum or testicular tunica on both sides of the scrotum to cover the ventral side of the formed urethra to increase the level of tissue and avoid sutures Overlap, significantly reduce the occurrence of complications such as urinary fistula. Sixth, the surgical technique is not demanding, easy to master, good postoperative appearance, smooth urine. In this group, $93.3 \%$ of the external genitalia were satisfied with the appearance, and the obstructive urinary flow curve was only $12.5 \%$. It has two disadvantages. Firstly, There is a suture opening on the dorsal and ventral sides of the urethra, which need to be sutured carefully, and the thread knot in the urethra when sutured on the dorsal side, the thread should not be too long. The author generally keeps $1-1.5 \mathrm{~mm}$, and the absorption time of the absorbable thread should be within With in one month, otherwise the thread knot is too long and urinary is not smooth, some of the thread knot urinary scale will condense and urethral stones will appear. Secondly, The operation is not complicated, but requires skilled operation skills

The indications for surgery are: applicable to any severe proximal hypospadias, especially hypospadias accompanied by scrotal division and penile-scrotal translocation.

Intraoperative precautions: (1) The urethroplasty flap is a fascial flap pedicled at the urethral opening, and the blood supply of the flap must be protected; (2) When the new urethral flap is formed in a tubular shape, the thread knots outside, no tension, and the shape is formed Do not leak urine afterwards. (3) The anastomosis between the new urethral skin tube and the head of the penis is close to the ventral side, the new urethral opening will no longer be on the same side, and there will be no urethral stenosis. In short, the I-stage urethroplasty with continuous Y skin flap of penis and scrotum makes full use of and rarely destroys the natural anatomical and physiological structure of the original hypospadias. It is an ideal surgical method for proximal hypospadias.

\section{COMPETING FINANCIAL INTERESTS}

The authors declare no competing financial interests.

\section{Author Contributions}

J.C.Z and X.W.P. designed the experiments. J.C.Z., Y.F.C., Y.L.W., Y.L. and T.Q.H. analyzed the data. X.W.P. and J.C.Z. wrote the manuscript. J.C.Z., Y.F.C., Y.L., T.Q.H., Y.L.W., Y.E.Z and X.W.P. revised the manuscript. All authors approved the final version of the manuscript.

\section{Acknowledgments}


We thank all the patients and healthy volunteers who agreed to participate in the present study and all those who helped us successfully complete the research.

\section{Reference}

[1] K. onomura, H. akizaki, N. himoda, T. oyama, M. urakumo, and T. oyanagi, urgical repair of anterior hypospadias with fish-mouth meatus and intact prepuce based on anatomical characteristics. ur Urol 4 68-71.

[2] T. oyanagi, K. onomura, H. akizaki, I. akeuchi, and T. amashita, xperience with one-stage repair of severe proximal hypospadias: operative technique and results. ur Urol 4 06-10.

[3] Y. ayashi, and Y. ojima, urrent concepts in hypospadias surgery. nt J Urol 5 51-64.

[4] H. mir, V.R. ayanthi, K. itahara, N. anismend, and S.A. off, odification of the Koyanagi technique for the single stage repair of proximal hypospadias. Urol 64 73-5; discussion 976.

[5] L. ang, G. uang, L. eng, Y. uang, X. a, Y. hang, M. uan, J. hang, and L. uang, New Modification of the Koyanagi Technique for the One-stage Repair of Severe Hypospadias. rology 3 75-9.

[6] Y. ugita, S. anikaze, K. oshino, and F. amamichi, evere hypospadias repair with meatal based paracoronal skin flap: the modified Koyanagi repair. Urol 66 051-3.

[7] A. ook, A.E. houry, C. eville, D.J. agli, W.A. arhat, and J.L. ippi Salle, multicenter evaluation of technical preferences for primary hypospadias repair. Urol 74 354-7, discussion 2357.

[8] S.A. Amukele, A.C. Weiser, J.A. Stock, and M.K. Hanna, Results of 265 consecutive proximal hypospadias repairs using the Thiersch-Duplay principle. J Urol 172 (2004) 2382-3.

[9] L.H. Braga, J.L. Pippi Salle, A.J. Lorenzo, S. Skeldon, S. Dave, W.A. Farhat, A.E. Khoury, and D.J. Bagli, Comparative analysis of tubularized incised plate versus onlay island flap urethroplasty for penoscrotal hypospadias. J Urol 178 (2007) 1451-6; discussion 1456-7.

[10] L.N. Reddy, One-stage repair of hypospadias. Urology 5 (1975) 475-8.

[11] W. Snodgrass, and S. Yucel, Tubularized incised plate for mid shaft and proximal hypospadias repair. J Urol 177 (2007) 698-702. 

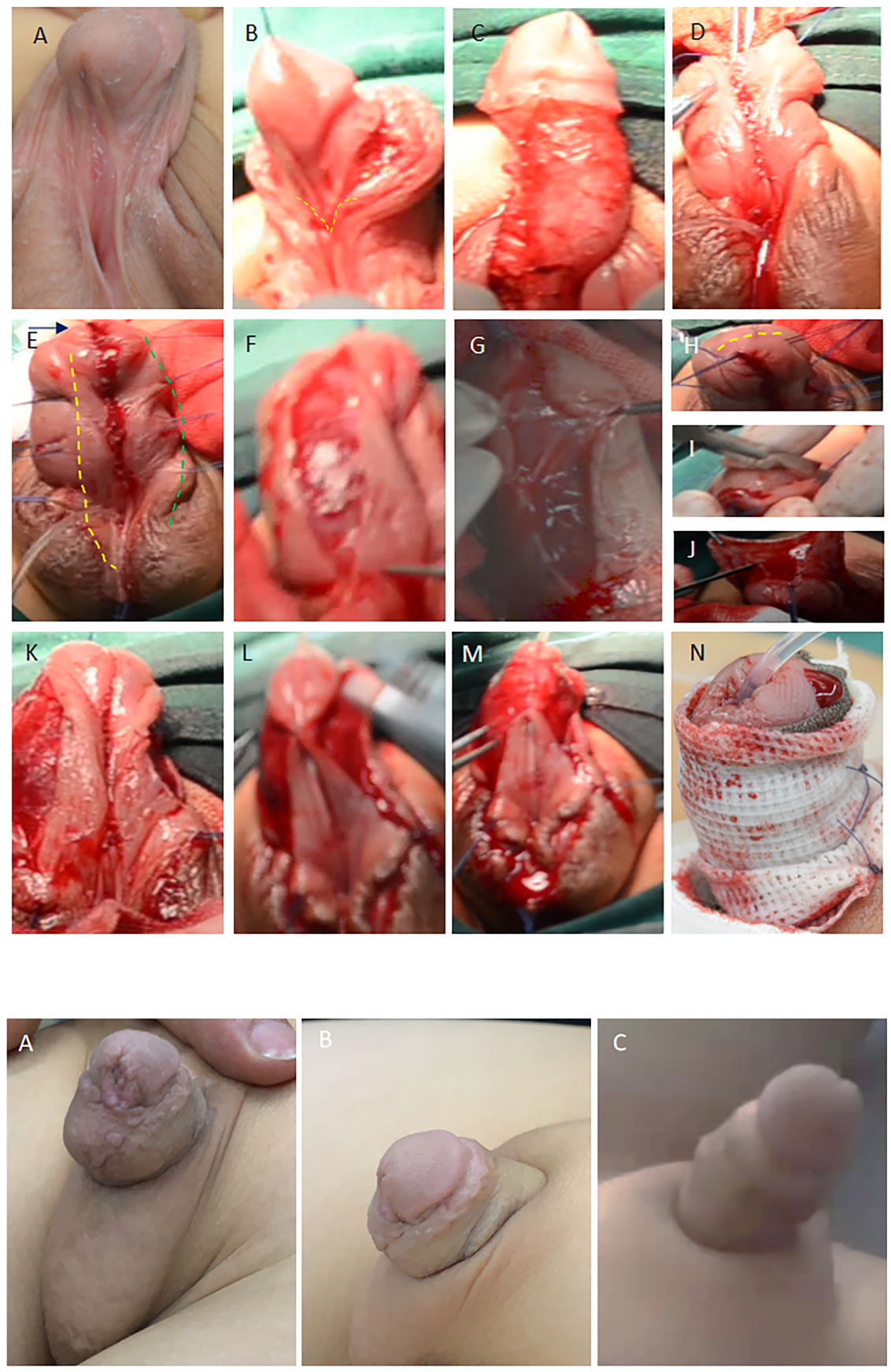